\title{
TRIGONOMETRIC SERIES WITH QUASI-MONOTONE COEFFICIENTS
}

\author{
S. M. SHAH
}

1. Introduction. In this note we extend the following Theorem $A$ of Chaundy and Jolliffe [3] and Theorems B and C of Boas [2].

Theorem A. Suppose that $b_{n} \downarrow 0$. A necessary and sufficient condition that the series

$$
S(x)=\sum_{1}^{\infty} b_{n} \sin n x
$$

should be uniformly convergent is that $n b_{n}=o(1)$.

TheOREM B. If $b_{n} \downarrow 0$ and $0 \leqq \gamma \leqq 1$, then

$$
x^{-\gamma} S(x) \in L(0, \pi)
$$

if and only if the series

$$
\sum_{1}^{\infty} n^{\gamma-1} b_{n}
$$

converges.

Theorem C. If $b_{n} \downarrow 0$ and $0<\gamma<1$ and

$$
C(x)=\sum_{1}^{\infty} b_{n} \cos n x,
$$

then $x^{-\gamma} C(x) \in L(0, \pi)$ if and only if (1.3) converges.

G. Sunouchi proved Theorems B and C by a different method [7] and Aljančić, Bojanić and Tomić [1], and S. O'Shea [6] have extended these theorems in different directions.

2. Statement of results. A sequence $\left(b_{n}\right)$ of nonnegative numbers is said to be quasi-monotone if

$$
b_{n+1} \leqq b_{n}(1+\alpha / n)
$$

for some constant $\alpha>0$ and all $n>n_{0}(\alpha)[5 ; 8]$. An equivalent definition is that $b_{n} / n^{\beta} \downarrow 0$ for some $\beta>0$. We may suppose that $\alpha$ is an integer. Let $P(n)$ denote the number of terms $b_{k}$ such that $k \leqq n$,

Presented to the Society, January 23, 1961; received by the editors September 19. 1960 and, in revised form, March 8, 1961. 
$b_{k-1}<b_{k}$; and let $\phi(x)$ be any function, positive and nondecreasing for $x \geqq 1$, and such that

(i)

$$
\phi\left(x^{2}\right) \leqq c \phi(x)
$$

for $x>1$ and some positive constant $c$ and

(ii)

$$
\sum_{1}^{\infty} 1 / n \phi(n)<\infty .
$$

THEOREM 1. Let $\left(b_{n}\right)$ be quasi-monotone.

(a) If either

$$
b_{n}=o(1), \quad P(n)=O(n / \phi(n)),
$$

or

$$
\sum_{1}^{\infty} n^{-1} b_{n}
$$

is convergent, then

$$
\sum_{1}^{\infty}\left|b_{n}-b_{n+1}\right|
$$

is convergent.

(b) If $n b_{n}=o(1)$, then

$$
\sum_{p}^{\infty}\left|b_{n}-b_{n+1}\right| \leqq \frac{(4 \alpha+1)}{p} \max _{k \geqq p}\left(k b_{k}\right) .
$$

(c) If either

$$
P(n)=O(n / \phi(n) \log n)
$$

and (2.3) is convergent, or

$$
\sum_{1}^{\infty}(\log n / n) b_{n}
$$

is convergent, then

$$
\sum_{1}^{\infty}\left|b_{n}-b_{n+1}\right| \log n
$$

is convergent.

(d) If $0<\gamma \leqq 1$ and (1.3) is convergent, then

$$
\sum_{1}^{\infty}\left|b_{n}-b_{n+1}\right| n^{\gamma}
$$


is convergent.

THEOREM 2. Let $\left(b_{n}\right)$ be quasi-monotone. If either (2.2) holds or (1.3) is convergent for $\gamma=0$, then (1.1) and (1.4) are convergent for every $x$, save possibly $x=0$ in the case of (1.4).

THEOREM 3. Let $\left(b_{n}\right)$ be quasi-monotone. A necessary and sufficient condition that the series (1.1) should be uniformly convergent throughout any interval is that $n b_{n}=o(1)$.

THEOREM 4. Let $\left(b_{n}\right)$ be quasi-monotone.

(i) If $0<\gamma<1$, and (1.3) is convergent, then $x^{-\gamma} C(x) \in L(0, \pi)$.

(ii) ${ }^{1}$ If $0<\gamma \leqq 1$, and (1.3) is convergent, then $x^{-\gamma} S(x) \in L(0, \pi)$.

(iii) If (1.3) is convergent for $\gamma=0$, and (2.5) holds, then

$$
C(x) \text { and } S(x) \in L(0, \pi) \text {. }
$$

REMARKS. I. Let $b_{n} \geqq 0$ (not necessarily monotone). Then [2, pp. 219-220] $x^{-\gamma} S(x) \in L(0, \pi), 0<\gamma \leqq 1$ implies the convergence of ${ }^{1}(1.3)$ and $x^{-\gamma} C(x) \in L(0, \pi), 0<\gamma<1$ implies the convergence of (1.3).

II. If $S(x) \in L(0, \pi)$ is odd and (1.1) its Fourier series, then $\sum b_{n} / n<\infty\left[4\right.$, p. $30 ; 9$, p. 59]. Hence, if $\left(b_{n}\right)$ be quasi-monotone, then (2.4) is convergent, and (1.1) is convergent everywhere and uniformly convergent over the interval $0<\delta \leqq x \leqq 2 \pi-\delta$.

These remarks along with Theorem 4 give extensions of Theorems $\mathrm{B}$ and $\mathrm{C}$ for series with quasi-monotone coefficients. We show by an example that the condition (2.2) on $P(n)$ is best possible in the sense that if $\sum 1 / n \phi(n)=\infty$, then (2.4) may not converge.

3. Proof of Theorem 1. (a) Write $b_{n}=b(n)$ and let

$$
S(p, q)=\sum_{p}^{q}|b(n)-b(n+1)| .
$$

If $P(k)=O(1)$, then the convergence of $(2.4)$ follows easily and so we assume $\lim _{k \rightarrow \infty} P(k)=\infty$. Let $n_{1} \geqq \max \left(n_{0}, 2\right)$ be the least integer such that $b\left(n_{1}+1\right)>b\left(n_{1}\right) ; p_{1} \geqq 1$ the largest integer such that $b\left(n_{1}+t\right)$ $>b\left(n_{1}+t-1\right)$ for $t=1,2, \cdots, p_{1} ; n_{2}>n_{1}+p_{1}$ the least integer such that $b\left(n_{2}+1\right)>b\left(n_{2}\right)$ and so on. Then $n_{k} \uparrow \infty$. Let $n_{k} \leqq p<n_{k+1}$, $n_{t} \leqq q<n_{t+1}$. Then $S\left(n_{j}, \quad n_{j+1}-1\right)=\sum_{1}(b(n+1)-b(n))$ $+\sum_{2}(b(n)-b(n+1))$ where the summation in $\sum_{1}$ is given by $n_{j} \leqq n \leqq n_{j}+p_{j}-1$ and in $\sum_{2}$ by $n_{j}+p_{j} \leqq n \leqq n_{j+1}-1$. Hence

$$
S(p, q) \leqq 2 \sum_{k}^{t}\left\{b\left(n_{j}+p_{j}\right)-b\left(n_{j}\right)\right\}+b\left(n_{k}\right) .
$$

\footnotetext{
${ }^{1}$ Compare [6, p. 281].
} 
Denote by $A, A_{1}, \cdots$ positive constants which may depend on $\alpha$ and $c$. Then from (2.2)

$$
p_{1}+\cdots+p_{k} \leqq P\left(n_{k}+p_{k}\right)<A\left(n_{k}+p_{k}\right) / \phi\left(n_{k}+p_{k}\right) .
$$

Hence for all large $k, p_{k} \leqq n_{k}$ and so for $k=1,2, \cdots, P\left(n_{k}+p_{k}\right)$ $\equiv P_{k}<A_{1} n_{k} / \phi\left(n_{k}\right)$. Write $n_{j}=n, p_{j}=p$. Then from (2.1)

$$
b(n+p) / b(n) \leqq \Pi(p, n, \alpha)
$$

where

$$
\begin{aligned}
\Pi(p, n, \alpha) & =(1+\alpha / n) \cdots(1+\alpha /(n+p-1)) \\
& <(1+p / n)^{\alpha} \leqq 1+\alpha \cdot 2^{\alpha-1}(p / n) .
\end{aligned}
$$

Hence from (3.1), we have

$$
S(p, q)<\left(\max _{j \geqq k} b\left(n_{j}\right)\right)\left(1+A_{2} \sum_{j=k}^{t} p_{j} / n_{j}\right) .
$$

Now

$$
\sum_{j=k}^{t} p_{j} / n_{j}<A_{1}\left[\sum_{k}^{t} \frac{1}{\phi\left(n_{j}\right)}\left(1-n_{j} / n_{j+1}\right)+\frac{1}{\phi\left(n_{k}\right)}+\frac{1}{\phi\left(n_{t+1}\right)}\right]
$$

Since

$$
\begin{gathered}
\sum_{n_{j}}^{n_{j+1}-1} 1 / n \phi(n)>\frac{1}{\phi\left(n_{j+1}\right)}\left(1-n_{j} / n_{j+1}\right) \\
\sum_{1}^{\infty} \frac{1}{\phi\left(n_{j+1}\right)}\left(1-n_{j} / n_{j+1}\right)<\infty
\end{gathered}
$$

and hence

$$
\sum_{1}^{\infty} \frac{1}{\phi\left(n_{j}\right)}\left(1-n_{j} / n_{j+1}\right)
$$

is convergent, provided $n_{j+1} \leqq n_{j}^{2}$ for all large $j$. If $n_{j+1}>n_{j}^{2}$ for an infinity of $j$, then we define $n_{j}^{*}$ as follows:

$$
n_{1}^{*}=n_{1}, \quad n_{j}^{*}=\min \left(n_{j}, n_{j-1}^{* 2}\right),
$$

It is easily seen that $n_{j-1}^{*}<n_{j}^{*} \leqq n_{j-1}^{* 2}$ for $j \geqq 2$ and

$$
\frac{1}{\phi\left(n_{j}\right)}\left(1-n_{j} / n_{j+1}\right)<\frac{A_{3}}{\phi\left(n_{j}^{*}\right)}\left(1-n_{j}^{*} / n_{j+1}^{*}\right) .
$$

By the above argument 


$$
\sum\left\{1 / \phi\left(n_{j}^{*}\right)\right\}\left(1-n_{j}^{*} / n_{j+1}^{*}\right)<\infty,
$$

and hence (3.4) is convergent. From (3.2), (3.3) and the convergence of (3.4), the convergence of (2.4) follows. If (2.3) is convergent, then since $\{b(n) / n\}$ is quasi-monotone [5],b(n)=o(1). Further, from (2.1), (3.6) $\quad b(n+p)-b(n) \leqq \alpha\left\{\frac{b(n)}{n}+\cdots+\frac{b(n+p-1)}{n+p-1}\right\}$.

Hence from (3.1), the convergence of (2.4) follows.

(b) If $n_{k} \leqq p<n_{k}+p_{k}$, then

$$
S\left(p, n_{k+1}-1\right)=2 b\left(n_{k}+p_{k}\right)-b(p)-b\left(n_{k+1}\right),
$$

and if $n_{k}+p_{k} \leqq p \leqq n_{k+1}-1$, then

$$
S\left(p, n_{k+1}-1\right)=b(p)-b\left(n_{k+1}\right) .
$$

Let $\mu(n)=\max _{k \geq n}\left(k b_{k}\right)$. Then

$$
\begin{aligned}
S\left(n_{k+1}, n_{t+1}-1\right) & =\sum_{j=k+1}^{t}\left\{2 b\left(n_{j}+p_{j}\right)-b\left(n_{j}\right)-b\left(n_{j+1}\right)\right\} \\
& \leqq 2 \alpha \sum_{n_{k+1}}^{\infty}\{b(n) / n\}+b\left(n_{k+1}\right) .
\end{aligned}
$$

Since

$$
\sum_{m}^{\infty} b(n) / n \leqq 2 \mu(m) / m, \quad S(p, \infty) \leqq(4 \alpha+1) \mu(p) / p .
$$

(c) Let

$$
S(p, q)=\sum_{p}^{q}\left|b_{n}-b_{n+1}\right| \log n
$$

where as in part (a), $n_{k} \leqq p<n_{k+1}, n_{t} \leqq q<n_{t+1}$. Then

$$
\begin{aligned}
S(p, q)< & \sum_{2}^{n_{t}+1} b(n) /(n-1) \\
& +\sum_{j=1}^{t}\left\{b\left(n_{j}+p_{j}\right) \log \left(n_{j}+p_{j}\right)-b\left(n_{j}\right) \log n_{j}\right. \\
& \left.+b\left(n_{j}+p_{j}\right) \log \left(n_{j}+p_{j}-1\right)-b\left(n_{j+1}\right) \log \left(n_{j+1}-1\right)\right\} .
\end{aligned}
$$

Now since $\sum b(n) / n$ is convergent, $b(n)=o(1)[5]$ and so

$$
S(p, q)<O(1)+A_{4}\left[\sum_{j=1}^{t} p_{j} \log n_{j} / n_{j}+A_{\mathbf{b}}\right] .
$$


From (2.5) we have

$$
\begin{aligned}
& \sum_{j=1}^{t}\left(p_{j} \log n_{j}\right) / n_{j} \\
& \quad<A_{6}\left[\sum_{j=1}^{t} \frac{1}{\phi\left(n_{j}\right)}\left(1-\frac{\log n_{j+1}}{n_{j+1}} / \frac{\log n_{j}}{n_{j}}\right)+\frac{n_{t} \log n_{t+1}}{\phi\left(n_{t}\right) n_{t+1} \log n_{t}}\right] .
\end{aligned}
$$

If $n_{j+1} \leqq n_{j}^{2}$ for all large $j$, then the series

$$
\sum_{1}^{\infty} \frac{1}{\phi\left(n_{j}\right)}\left\{1-\frac{\log n_{j+1}}{n_{j+1}} / \frac{\log n_{j}}{n_{j}}\right\}
$$

is convergent. If $n_{j+1}>n_{j}^{2}$ for an infinity of $j$, we define $n_{j}^{*}$, as in (3.5), and then prove the convergence of (3.10). From (3.8), (3.9) and the convergence of (3.10), the convergence of (2.7) follows.

To prove the second part of (c), we observe that for $\theta=0,1$

$$
\begin{aligned}
b\left(n_{j}+p_{j}\right) \log \left(n_{j}+p_{j}-\theta\right)-b\left(n_{j}\right) & \log \left(n_{j}-\theta\right) \\
& <A_{7}\left[\sum_{t=0}^{p_{j-1}} \frac{b\left(n_{j}+t\right) \log \left(n_{j}+t\right)}{n_{j}+t}\right] .
\end{aligned}
$$

Hence from (3.7) and the convergence of (2.6), the convergence of (2.7) follows.

(d) We omit the proof of the convergence of (2.8) which is similar to the one used to prove the second part of (c).

4. Proof of Theorem 2. This follows from the convergence of (2.4). We note that both series (1.1) and (1.4) are uniformly convergent over the interval $0<\delta \leqq x \leqq 2 \pi-\delta$.

Proof of Theorem 3 . We need consider the interval $0 \leqq x \leqq \pi$. To prove that the condition is necessary, consider

$$
S(p, q, x)=\sum_{p}^{q} b(n) \sin n x,
$$

and let $p=[(1 / 2) q+1], x=\pi / 2 q$. Then $b(r) \geqq b(q) / \Pi$ where $p \leqq r<q$ and

$$
\Pi=\left(1+\frac{\alpha}{q-p}\right) \cdots\left(1+\frac{\alpha}{q-1}\right)<A_{8}, \quad q>3
$$

Hence

$$
\begin{aligned}
S(p, q, x) & \geqq b(q) A_{8}^{-1}\{\sin p x+\cdots+\sin q x\} \\
& >A_{8}^{-1} b(q)\left(\frac{q}{2}-1\right) \sin \left(\frac{\pi}{4}\right) .
\end{aligned}
$$


Since the series is uniformly convergent, $q b(q)=o(1)$. We now show that the condition is sufficient. Let $n b(n)<\epsilon$ for $n \geqq N(\epsilon)$ and let $\max \left(N, n_{1}\right) \leqq p<q$. If $x \leqq \pi / q$, then $|S| \leqq p x b(p)+\cdots+q x b(q)$ $<x q \epsilon \leqq \pi \epsilon$. If $x \geqq \pi / p$, then from Theorem 1(b)

$$
\begin{aligned}
|S| & \leqq \operatorname{cosec}(x / 2)\left\{\sum_{p}^{q}|b(n)-b(n+1)|+b(p)+b(q+1)\right\} \\
& \leqq p\left\{\frac{\mu(p)(4 \alpha+1)}{p}+\frac{2 \mu(p)}{p}\right\} \leqq(4 \alpha+3) \epsilon .
\end{aligned}
$$

If $\pi / p<x<\pi / q$ then, with $k=[\pi / x]$, we have

$$
\begin{aligned}
|S| & \leqq|S(p, k)|+|S(k+1, q)| \\
& \leqq x k \epsilon+\frac{\pi}{x}\left\{\frac{(4 \alpha+3) \mu(k+1)}{k+1}\right\} \leqq \epsilon\{\pi+4 \alpha+3\}
\end{aligned}
$$

and the theorem is proved.

Proof of Theorem 4. Boas [2] proved that if $b_{n}=o(1)$ and

$$
\sum|b(n-1)-b(n+1)| n^{\gamma}
$$

is convergent, then $x^{-\gamma} S(x) \in L(0, \pi)$ where $0<\gamma \leqq 1$, and $x^{-\gamma} C(x)$ $\in L(0, \pi)$ where $0<\gamma<1$. By Theorem $1(\mathrm{~d})$, the series $(2.8)$ and hence (4.1) are convergent. Further from the convergence of (1.3) we have $b_{n}=o(1)[5]$. Hence (i) and (ii) are proved. To prove (iii) we note that

$$
\int_{0}^{\pi} \frac{1-\cos n x}{x} d x<A_{0} \log n, \quad \int_{0}^{\pi} \frac{|\sin n x|}{x} d x<A_{9} \log n .
$$

Hence by the argument of Boas [2], $C(x)$ and $S(x) \in L(0, \pi)$ if

$$
\sum|b(n-1)-b(n+1)| \log n
$$

is convergent. From Theorem $1(\mathrm{c}),(2.7)$ and hence (4.2) are convergent and (iii) is proved.

5. Example. Let $\phi(x)$ be positive and nondecreasing for $x \geqq 1$, $\phi\left(x^{2}\right) \leqq c \phi(x)$ for $x>1$ and $\sum 1 / n \phi(n)=\infty$. Then there exists a quasimonotone sequence $\left(b_{n}\right)$ for which

$$
b_{n}=o(1), P(n)=O(n / \phi(n)) \text { and } \sum_{1}^{\infty}\left|b_{n}-b_{n+1}\right|=\infty .
$$

Let $p_{i}=1+i, q_{i}=[i \phi(i)]+1, i=1,2, \cdots$,

$$
\begin{aligned}
n_{1} & =2, \quad n_{i+1}=n_{i}+p_{i}+q_{i}, \quad i=1,2, \cdots, \\
S(n) & =\sum_{1}^{n} 1 / k \phi(k),
\end{aligned}
$$




$$
\begin{aligned}
b_{n} \equiv b(n)=1 / S\left(n_{1}\right) & \text { for } n=1, \\
b\left(n_{k}\right) & =1 / S\left(n_{k}\right) \\
b\left(n_{k}+t\right) & =b\left(n_{k}\right)\left(n_{k}+t\right) / n_{k} \quad \text { for } t=1,2, \cdots p_{k} ; k=1,2, \cdots, \\
b\left(n_{k}+p_{k}+t\right) & =b\left(n_{k+1}\right) \text { for } t=1, \cdots, n_{k+1}-n_{k}-p_{k}-1 ; k=1,2, \cdots,
\end{aligned}
$$

Then $\left(b_{n}\right)$ is quasi-monotone and conditions (5.1) are satisfied.

Added in proof. (i) In Theorem 1 (a), (c), it is not necessary to suppose that $\phi\left(x^{2}\right) \leqq c \phi(x)$. In fact it can be proved that if $\lambda_{n}>0$, $\uparrow$ for $n=1,2, \cdots, \phi(x)>0, \uparrow$ for $x \geqq \lambda_{1}, \int_{\lambda_{1}}^{\infty} d x /\{x \phi(x)\}<\infty$, then $\sum_{1}^{\infty}\left\{\lambda_{n} / \phi\left(\lambda_{n}\right)\right\}\left(\lambda_{n}^{-1}-\lambda_{n+1}^{-1}\right)<\infty$.

(ii) The following theorem can be proved by the argument of P. Szüsz (Acta Math. Acad. Sci. Hungar. 12 (1961), 215-220). Let $K$ be any arbitrary large positive number and suppose there exists a sequence $\left(n_{k}\right), k=1,2, \cdots$ of natural numbers with the following properties: (a) $n_{k+1} / n_{k}>K ; k=1,2, \cdots$, (b) $\sum_{1}^{\infty} a_{k}\left|\sin \pi n_{k} x\right|<\infty$ for some $x \neq 0, \neq 1, \cdots$, where $\left(a_{n}\right)$ is quasi-monotone. Then $\sum_{1}^{\infty} a_{k}<\infty$.

\section{REFERENCES}

1. S. Aljančit, R. Bojaniţ et M. Tomit,, Sur l'intégrabilité de certaines séries trigonometriques, Acad. Serbe Sci. Publ. Inst. Math. 8 (1955), 67-84.

2. R. P. Boas, Jr., Integrability of trigonometric series. III, Quart. J. Math. Oxford Ser. (2), 3 (1952), 217-221.

3. T. W. Chaundy and A. E. Jolliffe, The uniform convergence of a certain class of trigonometric series, Proc. London Math. Soc. (2), 15 (1916), 214-216.

4. G. H. Hardy and W. W. Rogosinski, Fourier series, Cambridge Univ. Press, Cambridge, 1944.

5. S. M. Shah, A note on quasi-monotone series, Math. Student 15 (1947), 19-24.

6. S. O'Shea, Note on an integrability theorem for sine series, Quart. J. Math. Oxford Ser. (2), 8 (1957), 279-281.

7. G. Sunouchi, Integrability of trigonometric series, J. Math. Tokyo 1 (1953), 99-103.

8. O. Szász, Quasi-monotone series, Amer. J. Math. 70 (1948), 203-206.

9. A. Zygmund, Trigonometrical series, Vol. 1, Cambridge Univ. Press, New York, 1959.

UNIVERSITY OF KANSAS 